\title{
The Role of NMDA Receptor-operated Calcium Channels in Persistent Nociception after Formalin-induced Tissue Injury
}

\author{
Terence J. Coderre ${ }^{1,2,3}$ and Ronald Melzack ${ }^{3}$ \\ 'Pain Mechanisms Laboratory, Clinical Research Institute of Montreal, "Département de Médecine, Universite de \\ Montréal, and ${ }^{3}$ Department of Psychology, McGill University, Montreal, Quebec, Canada
}

The contribution of intracellular calcium to central sensitization and persistent nociception in response to tissue injury in rats was examined following the subcutaneous injection of formalin into the hindpaw. Formalin injury-induced nociceptive behaviors were enhanced by intrathecal pretreatment with the calcium ionophore A23187 or the calcium channel agonist Bay-K8644. Conversely, formalin nociceptive responses were reduced by intrathecal pretreatment with the calcium chelator Quin 2 or the calcium channel antagonists verapamil and nifedipine. Each of these agents affected the tonic, but not the acute, phase of the formalin response. The enhancement in formalin nociceptive behavior in rats treated with L-aspartate or L-glutamate was reversed by combined pretreatment with the noncompetitive NMDA antagonist MK-801, but not by nifedipine or the nonNMDA excitatory amino acid antagonist 6-cyano-7-dinitroquinoxaline-2,3-dione. In rats not treated with excitatory amino acids, the analgesic effect of MK-801 was also significantly greater than that produced by nifedipine. Furthermore, combining nifedipine with MK-801 did not produce a significantly greater analgesic effect than MK-801 alone. The results suggest that central sensitization and persistent nociception following formalin-induced tissue injury are dependent on the influx of calcium through predominantly NMDA receptor-operated (and to a lesser extent voltage-gated) calcium channels.

Studies in the preceding article indicate that central sensitization and tonic nociceptive behavior associated with formalin-induced tissue injury depend, in part, on activity at the NMDA receptor. Formalin injury-induced nociceptive behaviors were enhanced by intrathecal pretreatment with L-glutamate or L-aspartate, and were reduced by intrathecal pretreatment with the NMDA antagonists 5-amino-phosphonovalerate and MK801. Formalin nociceptive responses were also enhanced, although to a lesser degree, by intrathecal pretreatment with NMDA. Although these data establish a role for EAAs in injury-

\footnotetext{
Reccived Nov. 27, 1991; revised Fcb. 17, 1992; acceptcd Apr. 15, 1992.

This work was supported by grants from the Medical Research Council of Canada (MT-1 1045) and Fonds de la Recherche en Santé du Québec (900051) to T.J.C., and a Natural Sciences and Engineering Research Council grant (A7891) to R.M.

Correspondence should be addressed to Terence J. Coderre, Ph.D., Director, Pain Mechanisms Laboratory, Clinical Research Institute of Montreal, Quebec, Canada H2W 1 R7.

Copyright (C) 1992 Society for Neuroscience $0270-6474 / 92 / 123671-05 \$ 05.00 / 0$
}

induced central sensitization, excitatory amino acids (EAAs) alone cannot account for the prolonged changes in central neural function that accompany tissue injury. Since the activation of NMDA receptors is likely to produce physiological effects that last at most milliseconds (depolarization) to seconds (windup), additional intracellular effects are likely involved in the production of long-term central sensitization associated with the tonic nociceptive response.

An important characteristic of the NMDA receptor is that its channels are permeable to $\mathrm{Ca}^{2+}$ as well as to $\mathrm{Na}^{+}$and $\mathrm{K}^{+}$. Voltage-clamp studies of cultured neurons injected with a calciumsensitive dye indicate that calcium can enter cells after NMDA receptor activation in a manner that is independent of voltagegated calcium channels (MacDermott et al., 1986). Increases in intracellular calcium after NMDA receptor activation have been proposed to initiate biochemical processes responsible for plasticity induced by EAAs in various systems (see Cotman and Monaghan, 1988). Indeed, the enhanced calcium entry through NMDA receptor-operated channels leads to the activation of several intracellular messengers. Increased calcium influx following NMDA receptor activation is associated with the activation of guanylate cyclase and formation of cGMP (Novelli et al., 1987), the release of arachidonic acid (Lazarewicz et al., 1988), the translocation and activation of protein kinase $\mathrm{C}$ (Vaccarino et al., 1987), and the increased expression of the c-fos proto-oncogene (Szekely et al., 1987). It has been proposed that persistent NMDA receptor activation leads to alterations in membrane permeability following the phosphorylation of substrate proteins by these various intracellular messengers (Monaghan et al., 1989). It is possible that these intracellular calciumdependent alterations in membrane permeability contribute to central sensitization following formalin-induced tissue injury.

The present study examined the contribution of increased intracellular calcium to the persistent behavioral nociceptive responses following subcutaneous formalin injection in rats. Initial experiments assessed the effect of agents that increase or decrease the availability or entry of calcium into the intracellular space on formalin-induced nociception. Later experiments assessed the relative importance of calcium influx through voltagegated or NMDA receptor-operated calcium channels on formalin-induced nociception, as well as on the enhanced formalin nociceptive responses in rats treated with L-aspartate and L-glutamate. The results provide evidence that calcium influx through NMDA receptor-operated (and to a lesser extent voltage-gated) calcium channels contributes to central sensitization and tonic nociception following subcutaneous formalin injection. 


\section{Materials and Methods}

The experiments were performed on $250-350 \mathrm{gm}$ Long Evans hooded rats (Charles River). For nociceptive testing, rats were given a subcutaneous injection of either a standard $50 \mu \mathrm{l}$ of $2.5 \%$ formalin or a smaller volume of a lower concentration of formalin $(30 \mu \mathrm{l}$ of $1.0 \%)$ into the plantar surface of one hindpaw. Rats were then placed in a $30 \mathrm{~cm} \times$ $30 \mathrm{~cm} \times 30 \mathrm{~cm}$ Plexiglas box with a mirror below the floor at a $45^{\circ}$ angle to allow an unobstructed view of the paws. Observations for the purpose of generating nociceptive scores began immediately after formalin injection and were continued for $50 \mathrm{~min}$. A nociceptive score was determined for each 5 min time block according to the scale outlined in the preceding article (Coderre and Melzack, 1992).

Formalin nociceptive responses were assessed in an observer-blind manner in 20 groups of rats, following intrathecal administration of agents affecting either EAA receptors or the levels of intracellular calcium. In the first experiment, nociceptive responses to a standard formalin injection were assessed in vehicle-treated control rats and rats that were administered either a calcium ionophore (A23187, $1 \mu \mathrm{g}$ ), a calcium chelator (Quin 2, $1 \mu \mathrm{g}$ ), a calcium channel agonist (Bay-K8644, $1 \mu \mathrm{g}$ ), a phenylalkylamine-typc calcium channcl antagonist (verapamil, $10 \mu \mathrm{g}$ ), or a dihydropyridine-type calcium channel antagonist (nifedipine, $10 \mu \mathrm{g}$ ). In the second experiment, rats pretreated with intrathecal saline, A23187 $(1 \mu \mathrm{g})$, or Bay-K8644 $(1 \mu \mathrm{g})$ were given the lower concentration of formalin to assess the possible contribution of a ceiling effect on pain scores in these groups of rats when given the standard formalin injection. In the third experiment, rats treated with either the EAA agonist $\mathrm{L}$-glutamate $(20 \mu \mathrm{g})$ or L-aspartate $(25 \mu \mathrm{g})$ were compared on a standard formalin test with rats treated with a combined treatment with L-glutamate $(20 \mu \mathrm{g})$ or L-aspartate $(25 \mu \mathrm{g})$ and either nifedipine $(10$ $\mu \mathrm{g}$ ), 6-cyano-7-dinitroquinoxaline-2,3-dione (CNQX; $10 \mu \mathrm{g}$ ) or MK$801(20 \mu \mathrm{g})$. MK-801 is a noncompetitive NMDA antagonist that blocks the influx of calcium through NMDA receptor-operated calcium channels; CNQX is a selective antagonist of AMPA/kainate receptors. Finally, nociccptive rcsponses to a standard formalin injection were assessed in MK-801-treated rats and rats given a combined treatment with MK-801 $(20 \mu \mathrm{g})$ and nifedipine $(10 \mu \mathrm{g})$. These groups were compared with the effects of nifedipine $(10 \mu \mathrm{g})$, by itself, described above. All agents were dissolved in $0.9 \%$ saline [except A23187, Quin 2, and nifedipine, which were dissolved in $10 \%$ alcohol, $1 \%$ dimethyl sulfoxide (DMSO) or $10 \%$ acetone, respectively, in $0.9 \%$ saline] and given $10-15$ min prior to testing as an intrathecal injection, in a $20 \mu \mathrm{l}$ volume. The vehicle control group in the first experiment consisted of six rats each treated with intrathecal saline, $10 \%$ ethanol, $1 \% \mathrm{DMSO}$, or $10 \%$ acetone in saline; no significant differences were found between these four vehicle treatments; consequently, they were collapsed into one vehicle control group. Intrathecal injections were given by lumbar puncture between the L4 and L5 vertebrae while rats were under brief ether anesthesia. None of the calcium channel agents produced any behavioral effects on their own. Formalin injections were given after the rats had fully recovered from the ether anesthesia. Rats were killed immediately following testing by overdose with chloral hydrate. The experimental protocol was approved by the institutional animal care committee.

\section{Results}

Figure $1 A$ illustrates the formalin nociceptive scores for control rats and rats treated with agents affecting calcium availability or influx. The vehicle control group exhibited a typical biphasic nociceptive response with high nociceptive responses in the first $5 \mathrm{~min}$, a reduction in nociceptive behavior for $5-10 \mathrm{~min}$, and a subsequent increased level of nociceptive responses that started $20 \mathrm{~min}$ after formalin and continued until the completion of testing at $50 \mathrm{~min}$. Nociceptive responses were significantly elevated, compared to control rats, in rats pretreated with the calcium ionophore A23187 or the calcium channel agonist BayK8644. Both treatments significantly elevated nociceptive responses between 5 and $15 \mathrm{~min}$ after formalin injection, while Bay-K8644 also increased scores between 30 and 50 min after formalin. Importantly, intrathecal administration of these agents that increase calcium availability or influx resulted in increased nociceptive responses only during the intermediate and late phases of the formalin response. Nociceptive responses during the early phase (the first 5 min time block) of the formalin test were unaffected, indicating that the treatments did not simply produce an additive hyperalgesic effect. Rather, it appears that agents that increase intracellular calcium shifted the tonic phase of the formalin test to an earlier time point, so that nociceptive responses during the normally quiet intermediate phase were more like those normally seen during the late phase of the formalin test, as well as increasing nociception during the late phase in the case of Bay-K8644.

The effects of A23187 and Bay-K8644 were also assessed in rats given a lower concentration of formalin to determine whether a ceiling effect may have prevented nociccptive responses from reaching their maximum level. This could explain, for example, why nociceptive scores for rats treated with A23187 were only elevated during the intermediate phase of the formalin test when nociceptive scores are typically quite low. When these agents were given to rats tested with a lower concentration of formalin, nociceptive responses were significantly elevated, with respect to the control group, for the majority of the testing period (excluding the acute phase during the first $5 \mathrm{~min}$ for both groups, and at the 35 and 40 min time points for Bay K-8644) (Fig. $1 B)$.

In contrast to the hyperalgesic effects of agents that increase intracellular calcium, those agents that decrease intracellular calcium produced a reduction in formalin nociceptive responses. Although the phenylalkylamine-type calcium channel antagonist verapamil produced a slight reduction in formalin nociceptive behaviors that reached significance at only one time point, the dihydropyridine-type calcium channel antagonist nifedipine produced a significant reduction that was sustained over a period between 10 and $25 \mathrm{~min}$ after formalin injection. Furthermore, the calcium chelator Quin 2 produced a significant reduction in formalin nociceptive responses throughout much of the intermediate and late phases of the formalin test (between 10 and $30 \mathrm{~min}$, as well as at the $40 \mathrm{~min}$ time period) (Fig. 1 $\mathrm{A}$ ). Once again, none of these agents affected formalin nociceptive responses during the acute early phase of the formalin test, indicating that lowering intracellular calcium reduces the development of tonic nociception in the late phase but does not produce a generalized analgesia throughout the entire test.

Figure $2 A$ illustrates the formalin nociceptive responses for rats treated either with $L$-aspartate alone or with L-aspartate combined with nifedipine, CNQX, or MK-801. By itself, $\mathrm{L}$-aspartate produced an elevation in formalin nociceptive responses that was reflected by the absence of low nociceptive responses during the intermediate phase (5-15 $\mathrm{min}$ ) after formalin injection. The elevation in formalin nociceptive responses produced by L-aspartate was unaffected by combined treatment with either the non-NMDA EAA receptor antagonist CNQX or the calcium channel antagonist nifedipine. Conversely, the L-aspartate-induced elevation in nociceptive responses was significantly reduced by combined treatment with the noncompetitive NMDA receptor antagonist MK-801. The nociceptive responses of L-aspartate-treated rats given MK-801 were significantly reduced between 5 and $20 \mathrm{~min}$ post-formalin, as well as during the 40 min time block.

Figure $2 B$ illustrates the formalin nociceptive responses for rats treated either with L-glutamate alone or with L-glutamate combined with nifedipine, CNQX, or MK-801. Like L-aspartate, $\mathrm{L}$-glutamate produced an elevation in nociceptive responses that was reflected by the absence of low nociceptive responses during the intermediate phase (5-15 min) after formalin injec- 
A
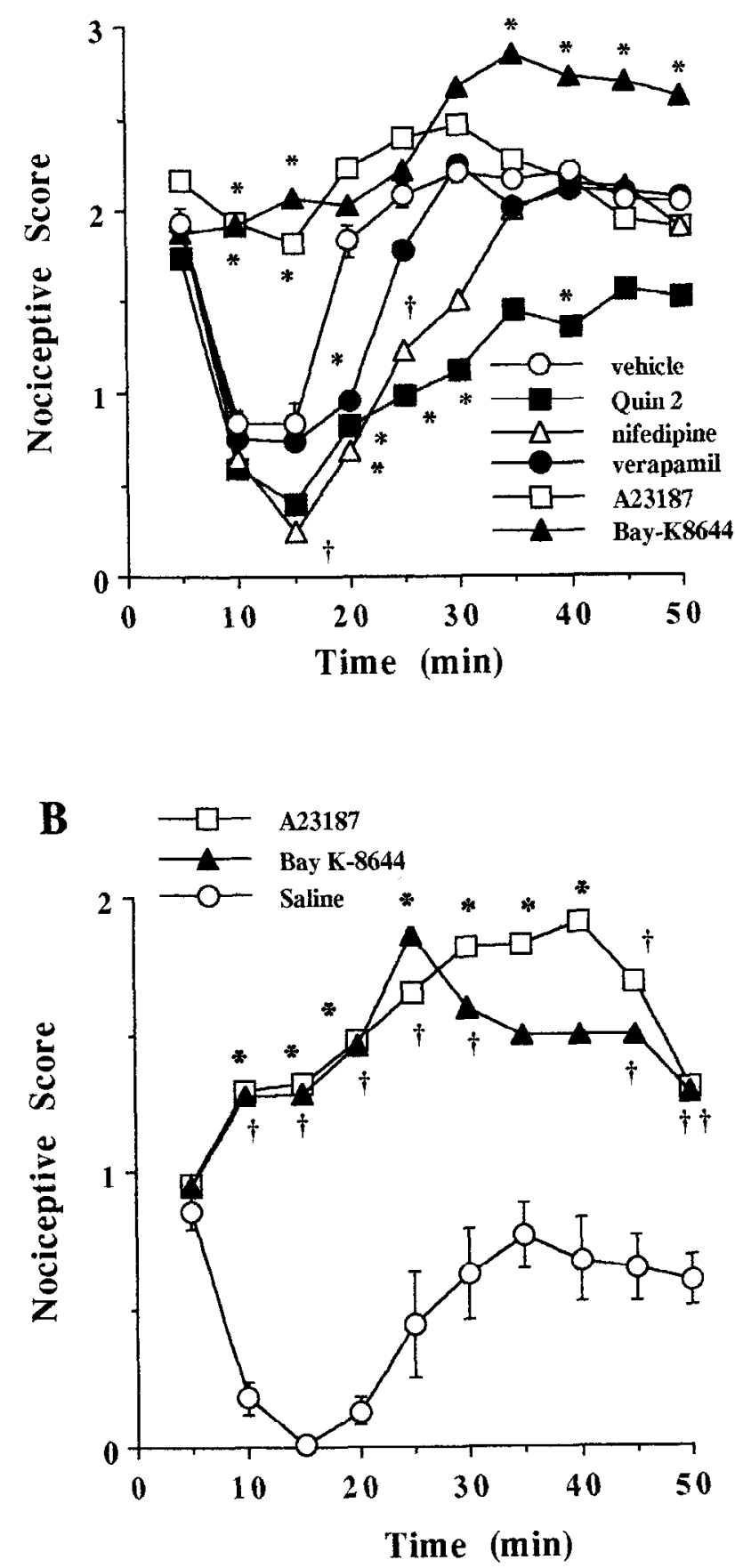

Figure 1. $A$, Nociceptive responses $( \pm$ SEM) induced by a standard formalin injection in rats pretreated with intrathecal vehicle, Bay-K8644 $(1 \mu \mathrm{g}), \mathrm{A} 23187(1 \mu \mathrm{g})$, Quin $2(1 \mu \mathrm{g})$, verapamil $(10 \mu \mathrm{g})$, or nifedipine $(10 \mu \mathrm{g}) . N=6$ for each group except the vehicle group, where $N=24$. Statistical analyses performed in all experiments were nonparametric multiple comparisons to the control group (Zar, 1984) subsequent to Kruskal-Wallis analysis of ranks. Significant differences from the vehicle control group in this and subsequent figures are indicated by the following symbols: $\dagger, p<0.05 ;{ }^{*}, p<0.01 . B$, Nociceptive responses $( \pm$ SEM) induced by a low concentration formalin injection in rats pretreated with intrathecal saline $(N=6)$, Bay-K8644 $(1 \mu \mathrm{g}, N=4)$, or A23187 (1 $\mu \mathrm{g}, N=4)$.
A

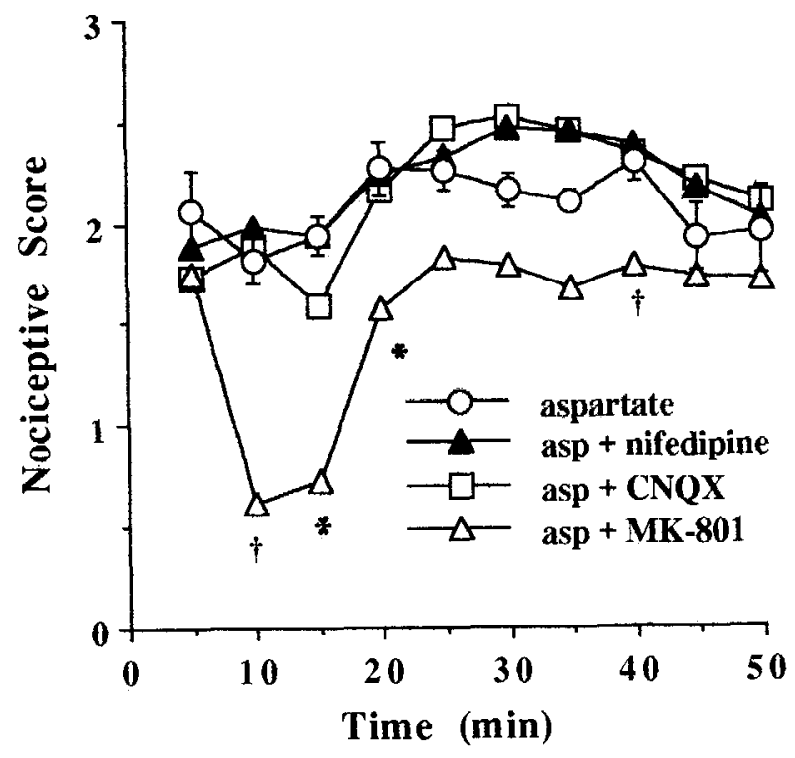

B

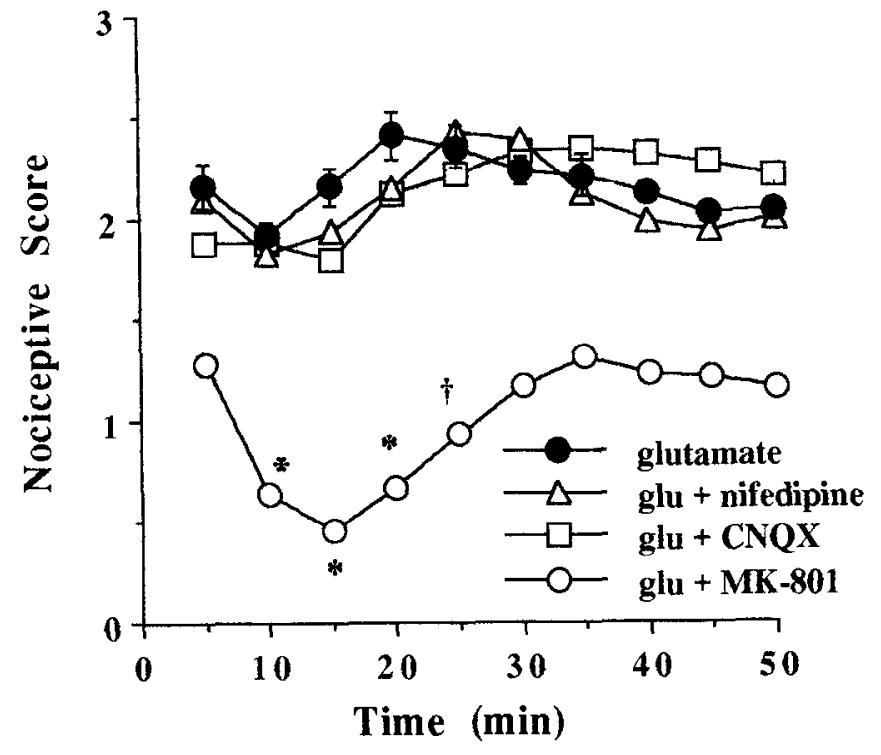

Figure 2. A, Nociceptive responses $( \pm \mathrm{SEM})$ induced by formalin in rats pretreated with intrathecal $\mathrm{L}$-aspartate $(20 \mu \mathrm{g})$, L-aspartate + nifedipine $(10 \mu \mathrm{g}), \mathrm{L}$-aspartate + CNQX $(5 \mu \mathrm{g})$, or L-aspartate + MK-801 $(20 \mu \mathrm{g}) . N=6$ for each group. $B$, Nociceptive responses $( \pm \mathrm{SEM})$ induced by formalin in rats pretreated with intrathecal L-glutamate $(20 \mu \mathrm{g})$, L-glutamate + nifedipine $(10 \mu \mathrm{g})$, L-glutamate + CNQX $(5 \mu \mathrm{g})$, or L-glutamate + MK-801 $(20 \mu \mathrm{g}) . N=6$ for each group, except L-glutamate + nifedipine $(N=5)$.

tion. The elevation in nociceptive responses produced by L-glutamate was also unaffected by combined treatment with either the non-NMDA EAA receptor antagonist CNQX or the calcium channel antagonist nifedipine. However, similar to L-aspartate, the L-glutamate-induced elevation in nociceptive responses was significantly reduced by combined treatment with the noncompetitive NMDA receptor antagonist MK-801. The nociceptive responses of L-glutamate-treated rats given $\mathrm{MK}-801$ were significantly reduced between 5 and $25 \mathrm{~min}$ post-formalin. 


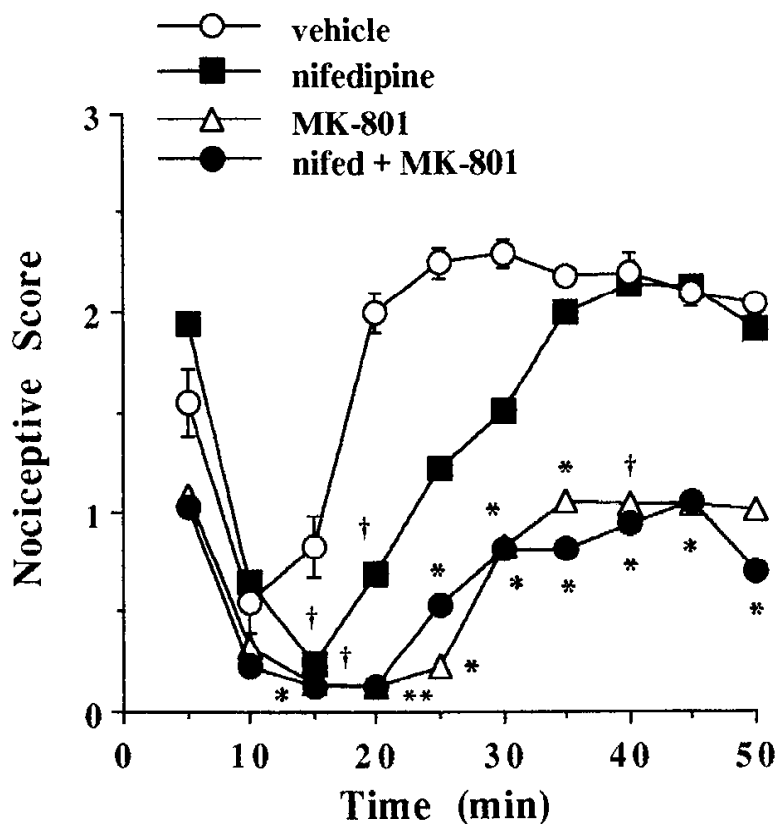

Figure 3. Nociceptive responses $( \pm$ SEM $)$ induced by formalin in rats pretreated with in trathecal vehicle, nifedipine $(10 \mu \mathrm{g})$, MK-801 $(20 \mu \mathrm{g})$, or nifedipine + MK-801. $N=12$ for the vehicle group; $N=6$ for all other groups. The nifedipine group is repeated from Figure $1 A$. The vchiclc group consisted of six rats cach given $0.9 \%$ saline or $10 \%$ acetone in $0.9 \%$ saline; no significant differences were observed between these two groups of rats.

Figure 3 illustrates the formalin nociceptive responses for rats treated with the non-competitive NMDA antagonist MK-801 and the calcium channel antagonist nifedipine given alone or combined. The NMDA antagonist MK-801, given by itself, produced a profound reduction in formalin nociceptive responses during both the intermediate and late phases of the formalin test (between 10 and 40 min post-formalin). This effect was greater than that produced by the calcium channel antagonist nifedipine. Although combining MK-801 with nifedipine produced effects that significantly reduced nociceptive responses (with respect to vchiclc) throughout the entire test, the combination did not significantly enhance the reduced formalin nociceptive responses found with MK-801 alone.

\section{Discussion}

The present study has demonstrated that formalin injury-induced nociceptive behaviors are enhanced by intrathecal treatment with a calcium ionophore and a calcium channel agonist, both of which increase the level of intracellular calcium. In addition, formalin nociceptive responses were decreased by intrathecal treatment with a calcium chelator and phenylalkylamine- and dihydropyridine-type calcium channel antagonists, each of which would decrease intracellular levels of calcium. Importantly, the effects of the agents were restricted to the tonic intermediate and late phases of the formalin test and did not affect the acute early phase. We suggest that cxccssive calcium influx is not critically involved in the transmission of nociceptive inputs in response to brief noxious stimuli, but rather is critically involved in mediating plasticity or central sensitization following tissue injury that leads to persistent nociception. This hypothesis is supported by previous results that indicate that brief, phasic nociceptive tests, such as the tail-flick and hotplate tests, are unaffected by $\mathrm{Ca}^{2+}$ (Harris et al., 1975; Chapman and Way, 1982), calcium chelators (Harris et al., 1975; BenSreti et al., 1983), or calcium channel antagonists (Benedek and Szikszay, 1984; Contreras et al., 1988), while tonic nociceptive tests, such as the acetic acid-induced writhing test, are sensitive to $\mathrm{Ca}^{2+}$ (Chapman and Way, 1982), which increases nociceptive responses, and calcium channel antagonists (Del Pozo et al., 1987), which reduce nociceptive responses.

Although these results implicate calcium influx as contributing to central sensitization and tonic nociception in the formalin test, they do not address the mechanism by which formalin-induced tissue injury produces an increase in intracellular calcium. Since the calcium chelator Quin 2 was more effective than verapamil or nifedipine at reducing formalin nociceptive responses, it is likely that calcium influx through channels other than phenylalkylamine- and dihydropyridine-type voltage-gated calcium channels is involved in this response. Other than this point, it is not clear from the results in Figure 1 whether the increased calcium is within presynaptic or postsynaptic cells, or whether calcium influx is increased through receptor-operated calcium channels or voltage-gated calcium channels. Given the results of the preceding article demonstrating a contribution of EAAs to formalin-induced nociceptive behaviors, we hypothesized that increases in intracellular calcium that enhance formalin responses are dependent on influx through NMDA receptor-operated calcium channels. To test this hypothesis, we first assessed the effects of agents affecting calcium influx on the enhanced formalin nociceptive responses in rats pretreated with the EAAs L-glutamate and L-aspartate. The results indicated that enhanced formalin nociceptive responses in rats pretreated with L-glutamate or L-aspartate were significantly reversed by combined treatment with a noncompetitive NMDA antagonist, which blocks NMDA receptor-operated calcium channels, but not by a calcium channel antagonist, which blocks voltage-gated calcium channels, or by a non-NMDA receptor antagonist. These results suggest that it is the NMDA receptor-operated calcium channels that are critical to the enhancement of formalin nociceptive responses by EAAs. It is noteworthy that MK-801 seemed to produce a greater reduction in formalin nociceptive responses in rats treated with $\mathrm{L}$-glutamate than in rats treated with L-aspartate. This may have resulted because although both aspartate and glutamate increase ligand binding to phencyclidine recognition sites where MK-801 acts (Loo et al., 1986; Fagg, 1987), glutamate is nine times more effective than aspartate in this respect (Loo et al., 1986).

Finally, to implicate further the contribution of NMDA receptor-operated calcium channels in central sensitization and tonic nociception following formalin-induced tissue injury, we compared the analgesic effects of the NMDA receptor-operated calcium channel antagonist MK-801, the voltage-gated calcium channel antagonist nifedipine, and a combination of these two agents in otherwise untreated rats. We have demonstrated that MK-801 is much more effective at reducing formalin nociceptive responses than is the voltage-dependent calcium channel antagonist nifedipine. This indicates that there is a greater contribution of NMDA receptor-operated calcium channels, as opposed to voltage-gated calcium channels, in the nociceptive response to formalin-induced tissue injury, although the latter probably make some contribution. Furthermore, combining MK801 with nifedipine did not add to the analgesic effect of MK- 
801 , suggesting that most of the effects of calcium influx on injury-induced central sensitization can be accounted for by NMDA receptor-operated calcium channels.

It is noteworthy that the calcium channel agonist Bay-K8644 produced an dramatic increase in formalin-induced nociceptive behaviors. Rats in this group (Fig. 1) exhibit a mean nociceptive score of 2.6 or above for the last 20 min of testing. This means that rats given this treatment spent greater than $85 \%$ of the time licking their injected hindpaw, at a point when nociceptive behaviors would normally be on the decline. Why docs this group exhibit such high levels of nociceptive behaviors? We suggest that the intracellular level of calcium is critical to the development of tonic nociceptive responses to formalin-induced tissue injury, and that normally the nociception induced by formalin injury depends predominantly on calcium influx through NMDA receptor-operated calcium channels. The treatment with Bay-K8644 enhances the influx of calcium through voltagegated calcium channels as well, and the further increase in calcium influx produces a further increase in nociception.

In previous studies, we have demonstrated that the tonic later phase of the nociceptive response to formalin injury depends on central sensitization induced by nociceptive inputs occurring during the brief early phase (Coderre et al., 1990). We also demonstrated in the preceding article that the late phase of the formalin test is maximally enhanced and perhaps accelerated following pretreatment with L-glutamatc, L-aspartatc, and a combination of NMDA and $(R, S)$ - $\alpha$-amino-3-hydroxy-5-methylisozazole-4-propionic acid hydrobromide (AMPA) or NMDA and trans- $(t)$-1-amino-1,3-cyclopentane dicarboxylic acid (Coderre and Melzack, 1992). Combined with the present data, which implicate a contribution to the formalin test of calcium influx through NMDA receptor-operated calcium channels, we have begun to describe a possible cellular mechanism for central sensitization following formalin-induced tissue injury. We propose that the intense activation of C-fiber afferents following formalin injury leads to a fast transmitter-mediated input to non-NMDA EAA receptors on spinal projection neurons, which underlies the early phase response in the formalin test. It is also expected that the depolarization associated with this fast transmitter activation of non-NMDA receptors (possibly the AMPA or kainate receptor) will reduce a $\mathrm{Mg}^{2+}$ blockade of NMDA receptor channels (Mayer et al., 1984; Nowak ct al., 1984), and mediate an NMDA-dependent windup in dorsal horn neurons (Davies and Lodge, 1987). The repetitive activation of the NMDA receptor would eventually cause an increased influx of calcium into the cell through NMDA receptor-operated calcium channels (MacDermott et al., 1986), and may mediate plastic changes within the cell by stimulating $\mathrm{Ca}^{2+} /$ calmodulin-dependent protein kinases (Miller and Kennedy, 1986). The increase in intracellular calcium, as well as activity at metabotropic EAA receptors, would lead to the production of second messengers, such as inositol trisphosphate and diacyclgycerol, which stimulate protein kinase C (PKC) (Vaccarino et al., 1987). By phosphorylating substrate proteins, the stimulation of $\mathrm{Ca}^{2+} /$ calmodulin-dependent protein kinases and PKC would alter ion channels and affect synaptic efficiency for a prolonged period of time, and thus may underlie central sensitization and the persistent nociceptive responsc following formalin-induced tissue injury.

\section{References}

Benedek G, Szikszay M (1984) Potentiation of thermoregulatory and analgesic effects of morphine by calcium antagonists. Pharmacol Res Commun 16:1009-1017.

Ben-Sreti MM, Gonzalez JP, Sewell RDE (1983) Effects of elevated calcium and calcium antagonists on 6,7-bemzomorphan-induced analgesia. Eur J Pharmacol 90:385-391.

Chapman DB, Way EL (1982) Modification of endorphin/enkephalin analgesia and stress-induced analgesia by divalent cations, a cation chelator and an ionophore. Br J Pharmacol 75:389-396.

Coderre TJ, Melzack R (1992) The contribution of excitatory amino acids to central sensitization and persistent nociception after formalin-induced tissue injury. J Neurosci 12:3665-3670.

Coderre TJ, Vaccarino AI, Melzack R (1990) Central nervous system plasticity in the tonic pain response to subcutancous formalin injection. Brain Res 535:155-158.

Contreras E, Tamayo L, Amigo M (1988) Calcium channel antagonists increase morphine-induced analgesia and antagonize morphine tolerance. Eur J Pharmacol 148:463-466.

Cotman CW, Monaghan DT (1988) Excitatory amino acid transmission: NMDA receptors and Hebb-type synaptic plasticity. Annu Rev Neurosci 11:61-80.

Davies SN, Lodge D (1987) Evidence for involvement of $N$-methylaspartate receptors in "wind-up" of class 2 neurones in the dorsal horn of the rat. Brain Res 424:402-406.

Del Pozo E, Caro G, Baeyens JM (1987) Analgesic effects of several calcium channel blockers in mice. Eur J Pharmacol 137:155-160.

Fagg GE (1987) Phencycline and related drugs bind to the activated $N$-methyl-D-aspartate receptor-channel complex in rat brain membranes. Neurosci Lett 76:221-227.

Harris RA, Loh HH, Way EL (1975) Effects of divalent cations, cation chelators and an ionophore on morphine analgesia and tolerance. J Pharmacol Exp Ther 195:488-498.

Lazarewicz JW, Wroblewski JT, Palmer ME, Costa E (1988) Activation of $N$-methyl-D-aspartate-sensitive glutamate receptors stimulates arachidonic acid release in primary cultures of cerebellar granule cells. Neuropharmacology 27:765-769.

Loo P, Braunwalder A, Lehmann J, Williams M (1986) Radioligand binding to central phencyclidine recognition sites is dependent on excitatory amino acid receptor agonists. Eur J Pharmacol 123:467468.

MacDermott AB, Mayer ML, Westbrook GL, Smith SJ, Barker JL, (1986) NMDA-receptor activation increases cytoplasmic calcium concentration in cultured spinal cord neurones. Nature 321:519-522.

Mayer ML, Westbrook GL, Guthric PB (1984) Voltage-dependent block by $\mathrm{Mg}^{2+}$ of NMDA responses in spinal cord neurones. Nature 309:261-263.

Miller SG, Kennedy MB (1986) Regulation of brain type II $\mathrm{Ca}^{21 /}$ calmodulin-dependent protein kinase by autophosphorylation: $\mathrm{a} \mathrm{Ca}^{2+}$ triggered molecular switch. Cell 44:861-870.

Monaghan DT, Bridges RJ, Cotman CW (1989) The excitatory amino acid receptors: their classes, pharmacology, and distinct properties in the function of the central nervous system. Annu Rev Pharmacol Toxicol 29:365-402.

Novelli A, Nicoletti F, Wroblewski JT, Alho H, Costa E, Guidotti A (1987) Excitatory amino acid receptors coupled with guanylate cyclase in primary cultures of cerebellar granule cells. J Neurosci 7:4047.

Nowak L, Bregestovski P, Ascher P, Hebert A, Prochiantz A (1984) Magnesium gates glutamate-activated channels in mouse central neurones. Nature 307:462-465.

Szekely AM, Barbaccia ML, Costa E (1987) Activation of specific glutamate receptor subtypes increases c-fos proto-oncogene expression in primary cultures of neonatal rat cerebellar granule cells. Neuropharmacology 26:1779-1782.

Vaccarino F, Guidotti A, Costa E (1987) Ganglioside inhibition of glutamate-mediated protein kinase $\mathrm{C}$ translocation in primary cultures of cerebellar neurons. Proc Natl Acad Sci USA 84:8707-8711.

Zar JH (1984) Biostatistical analysis. Englewood Cliffs, NJ: Prentice Hall. 